\title{
Does Conservation of Energy Hold in Atomic Processes?
}

\author{
By Prof. P. A. M. Dirac, F.R.S.
}

\section{$\mathrm{T}$} HE strict validity of the law of conservation of energy in atomic processes was not seriously questioned until 1924, when Bohr, Kramers and Slater, in order to make progress with the serious conflict then existing between the wave and corpuscular aspects of light, put forward a theory denying it'. This theory (which we shall refer to as the B.K.S. theory) postulates that a field of radiation is continually emitted by an atomic system in an excited state, instead of being emitted only when the system makes a transition to a state of lower energy. This field of radiation falling on a second atom gives it a probability of making a transition to a state of higher energy, provided the frequency of the radiation is suitable. The theory makes no coincidences between the occurrence of transitions of the second atom to states of higher energy and the occurrence of transitions of the first atom to states of lower energy, but apart from this question of coincidences, it gives results in agreement with those of other theories of radiation. Thus the new theory gives no conservation of energy for individual atomic processes, though it gives statistical conservation when large numbers of atomic processes take place.

Soon after the new theory was put forward, its predictions with regard to scattering of radiation by electrons were put to experimental test by Bothe and Geiger ${ }^{2}$ and by Compton and Simon ${ }^{3}$. The results in both cases were unfavourable to the new theory, and supported conservation of energy. Shortly after that, the new quantum mechanics was discovered by Heisenberg and by Schrödinger, and was developed to provide an escape from the difficulties of the conflict between waves and particles without departing from conservation of energy. Thus the B.K.S. theory was found to be in disagreement with experiment and was no longer required by theoretical considerations, and it was therefore abandoned.

The situation has now been changed by some recent experimental work of $R$. Shankland ${ }^{4}$. Shankland's experiments have been carried out with the greater accuracy which ten years of development of technique have made possible, and his results do not confirm those of the earlier experimenters. On the contrary, they are in disagreement with conservation of energy and require for their explanation something on the lines of the B.K.S. theory. Thus physics is now faced with the prospect of having to make a drastic change in its fundamentals, a change involving the giving up of some of its principles which have been most strongly relied on (conservation of energy and momentum), and the establishment in their place of the B.K.S. theory or something similar. We shall see, however, on closer considera. tion, that a large part of the present theoretical structure, in fact all the best part of it, may reasonably be retained, and thus the theoretical physicist need not be at all perturbed by Shank. land's results.

Further evidence about the conservation laws is provided by the modern work in nuclear physics. It is found that those nuclear processes in which only heavy particles (protons, neutrons, etc.) are concerned, and in which, consequently, all the velocities are small compared with the velocity of light, ean be accounted for fairly well on the basis of conservation of energy and momentum. On the other hand, processes involving an electron or positron, which is shot out with a large velocity, of the order of the velocity of light, seem to be in disagreement with conservation of energy, and a new unobservable particle, the neutrino, has been specially postulated by some investigators, in an attempt formally to preserve conservation of energy by assuming the unobservable particle to carry off the balance.

The above experimental results suggest that we take as the starting-point in our reformulation of atomic theory the assumption that energy and momentum are conserved in atomic processes in which the velocities concerned are all small com. pared with the velocity of light, but are not in general conserved in processes involving large velocities, including radiative processes. This assumption is all the more plausible on account of the fact that the present quantum mechanics, with its conservation of energy and momentum, forms a satisfactory theory only when applied non-relativistically, to problems involving small velocities, and loses most of its generality and beauty when one attempts to make it relativistic. In this way, we see that we can retain the whole of the present non-relativistic quantum mechanics, and we see the need for a profound alteration in current theoretical ideas, involving a departure from the conservation laws, before we can hope to get a satisfactory relativistic quantum mechanics.

Radiative processes are included among the large-velocity processes because of the large 
velocities of the light-quanta. According to the above assumption, the conservation laws should not apply for them, and the present comprehensive theory of them, the so-called quantum electrodynamics, must be given up. There exists, however, a primitive theory of radiation in quantum mechanics, namely, the theory which treats the electro-magnetic field classically as an external perturbing force, and this theory may be retained. This theory gives information about the probability of atoms and electrons making specified quantum jumps under the influence of radiation, but gives no information at all about the reaction of the atoms and electrons on the-radiation, and is thus equally consistent with the conservation laws and with the B.K.S. assumptions. This primitive theory of radiation gives no spontaneous emission, but by supplementing it with Einstein's laws of radiation and the B.K.S. assumptions, we can get a fairly complete account of all the elementary radiation phenomena, an account which is in satisfactory agreement with experiment and which is unsatisfactory only because of the lack of unity of its basic assumptions.

In retaining the non-relativistic quantum mechanics and the primitive theory of radiation with unquantised fields, we retain almost all the valuable part of present-day quantum theory. We may include in what we retain the theory of the spinning electron moving in a given electromagnetic field, and the Klein-Nishina formula. The only important part that we give up is quantum electrodynamics. Since, however, the only purpose of quantum electrodynamics, apart from providing a unification of the assumptions of radiation theory, is to account for just such coincidences as are now disproved by Shankland's experiments, we may give it up without regretsin fact, on account of its extreme complexity, most physicists will be very glad to see the end of it.

\footnotetext{
${ }^{1}$ Phil. Mag., 47, 785 (1924), or Z. Phys., 24, 69 (1924).

Z. Phys., 32, 639 (1925).

4 Phys. Rev., 49, 8 (1936). See Nature, 137, 241 (1936).
}

\section{Stephen Gray: The First Copley Medallist}

$\mathrm{O}^{\mathrm{N}}$ either February 15 or February 25, 1736, two hundred years ago, Stephen Gray, one of the earliest British electricians, died at the age of eighty-three years in Charterhouse, London, of which he had been an inmate for seventeen years. He had been nominated as a Brother in 1718 by George, Prince of Wales, but the entry in the records relating to his admission runs: "Stephen Gray for ye Prince, John Gwynn for ye Archbishop, John Cox for Earl Cowper and John Allen for ye Duke of Buckingham sworn at ye hospital before a Committee 24 June 1719."

Gray was then about sixty-six years of age, and though it is known that for a long time be had been devoted to physical science and astronomy, it is for the experiments he made in his room and in the courts of Charterhouse when he was over seventy that he will always be remembered. There is a short notice of him by James Burnley in the "Dictionary of National Biography" and the Philosophical Transactions contain a number of his papers, but the best account of his activities was given in a lecture delivered in the Great Hall, Charterhouse, by Dr. (afterwards Sir) Benjamin Ward Richardson (1828-96) on January 22, 1874, a report of which appeared in NATURE a week later.

Dr. Richardson had evidently studied Gray's papers in the Philosophical Transactions, and during the lecture he repeated many of Gray's experiments. Gray apparently belonged to Kent and, said Dr. Richardson, he is first discovered at Canterbury in 1692 making observations of a mock sun on February 6. Four years later he was making a water microscope, and in 1698 a microscope with a micrometer for measuring accurately the height of the mercury in a barometer. He studied the fossils of Reculver Cliff in Kent, made observations of sunspots and watched the solar eclipse of 1706, having at Canterbury a well-equipped observatory.

After 1706 Gray is lost sight of until he is recommended as a pensioner for Charterhouse. His first electrical paper in the Philosophical Transactions, entitled "An Account of Some New Electrical Experiments", appeared in 1720, and for the next ten or twelve years he devoted himself entirely to electricity. His apparatus consisted of such things as feathers, hair, silk, wool, soap bubbles, resin slabs, beeswax and cubes of oak; and he made observations on the electricity of the human body. Perhaps his greatest achievement was transmitting electricity through long lengths of pack-threads. In the grounds of the mansion of his friend Granville Wheler, Otterden House, Faversham, on July 14, 1729, he transmitted electricity through a pack-thread line $650 \mathrm{ft}$. long suspended by silk from poles, thus anticipating 